\title{
Home-based step training using videogame technology in people with Parkinson's disease: a single-blinded randomised controlled trial
}

Clinical Rehabilitation 2018, Vol. 32(3) 299-31। (C) The Author(s) 2017 Reprints and permissions: sagepub.co.uk/journalsPermissions.nav DOI: $10.1177 / 0269215517721593$ journals.sagepub.com/home/cre @SAGE

\author{
Jooeun Song ${ }^{1,2}$, Serene S Paul',3, Maria Joana D Caetano4, \\ Stuart Smith ${ }^{5}$, Leland E Dibble ${ }^{6}$, Rachelle Love', \\ Daniel Schoene ${ }^{7}$, Jasmine C Menant ${ }^{4}$, Cathie Sherrington ${ }^{3}$, \\ Stephen R Lord ${ }^{4}$, Colleen G Canning' ${ }^{\prime}$ and Natalie E Allen'
}

\begin{abstract} Parkinson's disease.

Design: A single-blinded randomised controlled trial.

\footnotetext{
'Faculty of Health Sciences, The University of Sydney, Sydney, NSW, Australia

${ }^{2}$ Graduate School of Health, University of Technology Sydney, Sydney, NSW, Australia

${ }^{3}$ Musculoskeletal Health Sydney, School of Public Health, The University of Sydney, Sydney, NSW, Australia

${ }^{4}$ Falls, Balance and Injury Research Centre, Neuroscience Research Australia, The University of New South Wales,
} Sydney, NSW, Australia
\end{abstract}

Objectives: To determine whether 12-week home-based exergame step training can improve stepping performance, gait and complementary physical and neuropsychological measures associated with falls in

Setting: Community (experimental intervention), university laboratory (outcome measures).

Subjects: Sixty community-dwelling people with Parkinson's disease.

Interventions: Home-based step training using videogame technology.

Main measures: The primary outcomes were the choice stepping reaction time test and Functional Gait Assessment. Secondary outcomes included physical and neuropsychological measures associated with falls in Parkinson's disease, number of falls over six months and self-reported mobility and balance.

Results: Post intervention, there were no differences between the intervention $(n=28)$ and control $(n=25)$ groups in the primary or secondary outcomes except for the Timed Up and Go test, where there was a significant difference in favour of the control group $(P=0.02)$. Intervention participants reported mobility improvement, whereas control participants reported mobility deterioration-between-group difference on an II-point scale $=0.9(95 \%$ confidence interval: $-I .8$ to $-0.1, P=0.03)$. Interaction effects between intervention and disease severity on physical function measures were observed $(P=0.0 \mathrm{I}$ to

\footnotetext{
${ }^{5}$ Southern Cross University, Coffs Harbour, NSW, Australia ${ }^{6}$ Department of Physical Therapy, University of Utah, Salt Lake City, UT, USA

${ }^{7}$ Institute for Biomedicine of Ageing, Friedrich-Alexander University Erlangen-Nürnberg, Erlangen, Germany
}

Corresponding author:

Natalie E Allen, Faculty of Health Sciences, The University of Sydney, P.O. Box 170, Lidcombe, NSW 1825, Australia. Email: natalie.allen@sydney.edu.au 
$P=0.08)$ with seemingly positive effects for the low-severity group and potentially negative effects for the high-severity group.

Conclusion: Overall, home-based exergame step training was not effective in improving the outcomes assessed. However, the improved physical function in the lower disease severity intervention participants as well as the self-reported improved mobility in the intervention group suggest home-based exergame step training may have benefits for some people with Parkinson's disease.

\section{Keywords}

Parkinson's disease, exergames, stepping performance, home-based training

Received: I4 February 2017; accepted: 28 June 2017

\section{Introduction}

Most people with Parkinson's disease suffer falls as the disease progresses ${ }^{1}$ and this debilitating problem responds poorly to Parkinson's disease medications. ${ }^{2}$ Due to the common motor impairments associated with Parkinson's disease, these individuals have reduced ability to make quick and appropriately timed steps, a critical skill for maintaining balance and preventing falls. ${ }^{3}$

Research has shown that supervised step training is feasible and efficacious in people with Parkinson's disease. ${ }^{4-6}$ A recent large $(n=282)$ trial of supervised treadmill walking in a virtual reality environment showed an improvement in balance and gait and a reduction in falls following the intervention, suggesting that step training may be an important component of exercise interventions to reduce falls and improve mobility in people with Parkinson's disease. While fully supervised, centre-based step training programmes appear to be feasible and effective; ${ }^{4,5}$ they are difficult to implement broadly and to sustain in the long term. In contrast, exercise-based videogames (exergames) can be set up in participants' homes and delivered unsupervised, after initial supervised training to maximise safety while exercising. Such exergames have built-in motivating features and provide immediate feedback on performance, thereby enhancing their potential to provide engaging and sustainable exercise. ${ }^{7}$ This is particularly important as a recent qualitative study identified difficulty in maintaining motivation and the desire for feedback on performance as key factors influencing exercise participation in people with Parkinson's disease. ${ }^{8}$ Furthermore, a feasibility study where people with Parkinson's disease were supervised while using a commercially available stepping exergame reported that participants could play the game safely without physical assistance and found the game to be fun and easy to use. ${ }^{9}$ This form of step training therefore has the potential to provide engaging and relatively inexpensive exercise that people with Parkinson's disease could undertake at home at times convenient to them.

There is evidence that unsupervised homebased step training using a modified dance-based game (Stepmania) is acceptable to older people and people with multiple sclerosis. Furthermore, in these populations, it was effective in improving choice stepping reaction time and balance (i.e. postural sway on a compliant surface, alternate step test), thereby reducing fall risk. ${ }^{7,10}$ The aim of the present trial was to determine whether this exergame training programme, which requires participants to make appropriately timed step responses to visually displayed targets presented on a television screen, is also efficacious in people with Parkinson's disease. Therefore, we hypothesised that 12 weeks of this exergame step training would (1) improve stepping performance and gait; (2) improve leg muscle power, mobility, 
simple reaction time, cognition, freezing of gait and fear of falling; and (3) reduce falls in people with Parkinson's disease.

\section{Methods}

The study comprised a two-arm, parallel, singleblinded randomised controlled trial and was prospectively registered with the Australian and New Zealand Clinical Trials Registry (ACTRN12613000688785).

Community-dwelling people with Parkinson's disease were recruited from metropolitan Sydney, Australia, through Parkinson's disease support groups and neurology clinics. Participants were included if they had been diagnosed with idiopathic Parkinson's disease by a neurologist and were living in the community, aged 40 years or over, were able to walk unaided for $\geq 30 \mathrm{~m}$ and their Parkinson's disease medication was stable for at least two weeks. They were excluded if they had substantial cognitive impairment (defined as a Mini-Mental State Examination Score $<24)^{11}$ or medical conditions which would preclude or interfere with physical assessment or stepping training. Demographic characteristics (age, gender, height, weight, mental status, Parkinson's disease duration, Parkinson's disease severity as determined by the Movement Disorders Society-sponsored version of the Unified Parkinson's Disease Rating Scale Part III, ${ }^{12}$ previous falls and freezing of gait) were collected by trained physiotherapists in order to describe the sample. All demographic information was gathered in the "on" medication state. The University of Sydney Human Research Ethics Committee approved this study and all participants gave written informed consent before data collection began.

Participants were randomly allocated into either the intervention or the control group within one day of the initial measurement session. The trial manager emailed the allocating researcher, who was located offsite and was not involved in recruitment, intervention or outcome assessment. The random allocation was conducted using a computer-generated table with randomly permuted blocks, and the result was communicated by email to the trial manager and the physiotherapist providing the training.

Participants in the intervention group were taught to perform the exergame in their home by an experienced physiotherapist. Participants were instructed to perform the exergame for a minimum of 15 minutes, three times a week for 12 weeks, while their usual Parkinson's disease medications were working optimally (i.e. during the "on" phase). The exergame was a modified version of the open-source Dance Dance Revolution "Stepmania" game (www.stepmania. com) which has been described in detail elsewhere. ${ }^{7}$

In brief, participants in the intervention group were provided with a small computer to connect to their television or monitor and a custom-made step mat. The step mat had six arrows (right, left, two fronts and two backs) as well as two central stance panels to indicate the position to initiate steps and return to after completing them. Participants were instructed to stand on the central stance panels and step on the appropriate target arrow (right, left, right front or back and left front or back) to match the direction and timing (varying stimulus speed) of the arrows that were drifting from the bottom to the top of the television screen.

To increase the cognitive load, there were three different types of randomly presented targets which sought different responses to get a perfect score: (1) an arrow required participants to step on the corresponding arrow on the step pad at the moment the arrow matched a target on the screen; (2) a tailed arrow required the same stepping response, with the step held until the tail of the arrow disappeared; and (3) a bomb-shaped object required participants to inhibit their step response.

After each step, participants were given feedback in the form of a word in the centre of the screen (perfect, good, miss) and accrued points were presented on the screen after finishing each game. The duration of each game was 2-3 minutes and participants were given the option to play music embedded in the system while training, but the stepping sequence patterns were not synchronised with the rhythm of the music so that auditory cues were not given.

The step training game had four levels of difficulty: novice, easy, medium and hard. All participants started with the easy level at the first home 
visit. At the second home visit, the physiotherapist modified the level as appropriate based on participants' performance and then participants were instructed to progress or regress the level of difficulty as appropriate.

Participants received two initial home visits from the study physiotherapist to set up the exergame system and to be taught how to use it safely. Participants were provided with all equipment and a booklet containing safety precautions and instructions, as well as a log book for recording completed exercise and any adverse events. The safety precautions and use of the log book were discussed with each participant and they were informed about potential negative effects of exercise (e.g. muscle soreness). An additional home visit was provided at six weeks to monitor progress. Additionally, the study physiotherapist telephoned participants every two weeks to discuss safe use of equipment and progression of training and to address any issues related to the exercise programme.

The control group received no intervention as part of the trial. Both groups were asked to continue with their usual healthcare.

All outcomes (except for number of falls) were collected by experienced physiotherapists at baseline and at the end of the 12-week trial at a university laboratory, with each assessment session lasting approximately 2.5 hours. Measurements were conducted while participants were "on" their usual Parkinson's disease medication. To maintain blinding of assessors, participants were asked not to discuss any aspect of the trial during the assessments.

The primary outcome measures were stepping performance (choice stepping reaction time test) ${ }^{13}$ and gait (Functional Gait Assessment). ${ }^{14}$ The choice stepping reaction time test has been shown to be a strong predictor of falls in older adults. ${ }^{13}$ The custom-made step mat used for the training was also used for the test. Participants were asked to stand on the two centre panels. The configuration of the step panels was presented on a monitor in front of the participant. Participants were asked to make rapid step responses to target step panels in response to corresponding, randomly visual stimuli presented on the monitor and return to the centre panels. Six practice trials and 18 test trials were administered. The choice stepping reaction time performance was measured in milliseconds and subdivided into (1) reaction time (i.e. stimulus presentation to foot lift-off), (2) movement time (i.e. foot lift-off to step-down) and (3) total response time (i.e. sum of the reaction time and movement time). The Functional Gait Assessment is an ambulation-based balance test and has been shown to be reliable and valid for assessing balance in people with Parkinson's disease ${ }^{14}$ and to predict falls in community-dwelling older adults. ${ }^{15}$ Each item is scored on an ordinal scale, ranging from 0 (severe impairment) to 3 (normal) and the total score of 10 items (maximum score of 30) was calculated.

Secondary outcomes were physical and neuropsychological measures associated with falls in Parkinson's disease, number of falls over six months and self-reported mobility and balance. Hip abductor muscle power was measured in Watts for each leg using pneumatic variable resistance equipment (Keiser A420, Keiser Sports Health Equipment, Fresno, CA). Muscle power was measured by having the participant abduct the hip as fast as possible against six relative loads $(30 \%-80 \%$ of the one repetition maximum at $10 \%$ increments), with peak power defined as the highest power measure among all the lift loads. Additionally, muscle power was measured at low load $(35 \mathrm{~N}-$ equivalent to $30 \%$ of the one repetition maximum on average in people with Parkinson's disease). ${ }^{16}$ Peak power and power at low load measures were recorded as the average of both legs.

The Timed Up and Go test was performed twice at a comfortable, safe pace and timed using a stopwatch; the better score was used for data analysis. ${ }^{17}$ The Timed Up and Go test was also performed with participants undertaking a secondary cognitive task (i.e. counting backward by 3 from a randomly given number between 80 and 100).

Gait adaptability was assessed by measuring participants' ability to quickly alter their gait in response to obstacles and stepping targets appearing on a walkway. ${ }^{18}$ The velocity of the stride preceding the target/obstacle (averaged from successful trials) and target stepping accuracy (distance between the centre of the target and the centre of the 
foot) were measured using an electronic walkway (ZenoMetrics ${ }^{\circledR}$ mat/PKMAS software, v2011-2013, Havertown, PA, USA).

For the assessment of simple reaction time, participants were seated at a table and asked to press a button of a modified computer mouse using the index finger of their dominant hand as quickly as possible when a light stimulus appeared. ${ }^{19}$ Five practice trials were undertaken, followed by 10 experimental trials, with the average time of the experimental trials calculated in milliseconds.

Global cognitive function was assessed using the Montreal Cognitive Assessment (version 7.1.), with the total score adjusted for education level (i.e. an additional point for individuals with 12 years or less of formal education). ${ }^{20}$ Processing speed and executive function were assessed with the Trail Making Test parts A and B. ${ }^{21}$ Participants were instructed to connect consecutive circled numbers for the Trail Making Test-part A and to connect numbers and letters in an alternating sequence for the Trail Making Test-part B, as quickly as possible without lifting the pen from the paper. If participants made an error they were informed immediately and allowed to correct it. The total time to complete each part was measured in seconds with the test time capped at 5 minutes.

The New Freezing of Gait Questionnaire was used to measure freezing of gait. ${ }^{22}$ Participants' concern about falling was determined with the Fall Efficacy Scale-International Questionnaire. ${ }^{23}$ Falls were recorded prospectively for six months via monthly falls diaries. Participants received a monthly phone call to confirm any reported falls and to provide reminders to mail the diaries when required. ${ }^{24}$ Adverse events and medications were also monitored and recorded throughout the study via these standardised calls. Each participant's perception of any overall change in his or her mobility and balance over the 12 -week trial period was assessed using a standard 11-point scale, ranging from -5 (very much worse) through 0 (unchanged) to +5 (very much improved). ${ }^{25}$

Between-group comparisons of final test performance were made using General Linear Models (analysis of covariance) controlling for baseline performance. An independent samples $t$-test (two-sided tail) was used to compare groups on participants' perception of overall change during the trial period. Fall rates in each group were compared using incidence rate ratios from a Poisson Inverse Gaussian regression model with the days of follow-up included as an exposure term in the model. ${ }^{24} \mathrm{~A}$ post hoc analysis was undertaken to determine whether there was a differential effect of the intervention according to disease severity on physical function outcomes. The sample was dichotomised at the median to produce lower (Movement Disorders Society-sponsored version of the Unified Parkinson's Disease Rating Scale Part III $\leq 32, n=30$ ) and higher (Movement Disorders Society-sponsored version of the Unified Parkinson's Disease Rating Scale Part III $>32, n=30$ ) disease severity subgroups, and a group by severity interaction term was entered into the analyses. An intentionto-treat approach was used for all analyses.

A sample size of 21 participants per group was calculated as being required, based on a Type 1 error of 0.05 , a Type 2 error of 0.20 (statistical power of $80 \%$ ), considering a $20 \%$ between-group difference in a different type of choice stepping reaction time test used in a previous study (assuming control group mean for completing 12 steps $=37 \mathrm{sec}$ onds, SD: 13) in people with Parkinson's disease. ${ }^{26}$

\section{Results}

The flow of participants through the trial is shown in Figure 1. A total of 60 people consented to participate in the trial and were randomly allocated to either the intervention $(n=31)$ or the control $(n=29)$ group. The demographic characteristics of the participants at baseline appeared to be similar in each group (Table 1). The daily levodopa equivalent dose $\mathrm{e}^{27}$ was similar between groups at baseline (a mean of $668 \mathrm{mg}$, SD of 405 for the intervention group, and a mean of $757 \mathrm{mg}$, SD of 498 for the control group) and at post-test (a mean of $646 \mathrm{mg}$, SD 352 for the intervention group, and a mean of $784 \mathrm{mg}$, SD of 614 for the control group) with little change in the dose during the trial.

Participants in the intervention group kept log books of their stepping exercise. Of the prescribed 36 exercise sessions, participants completed a mean of 31 (SD: 13) sessions, that is, $86 \%$. Six participants 


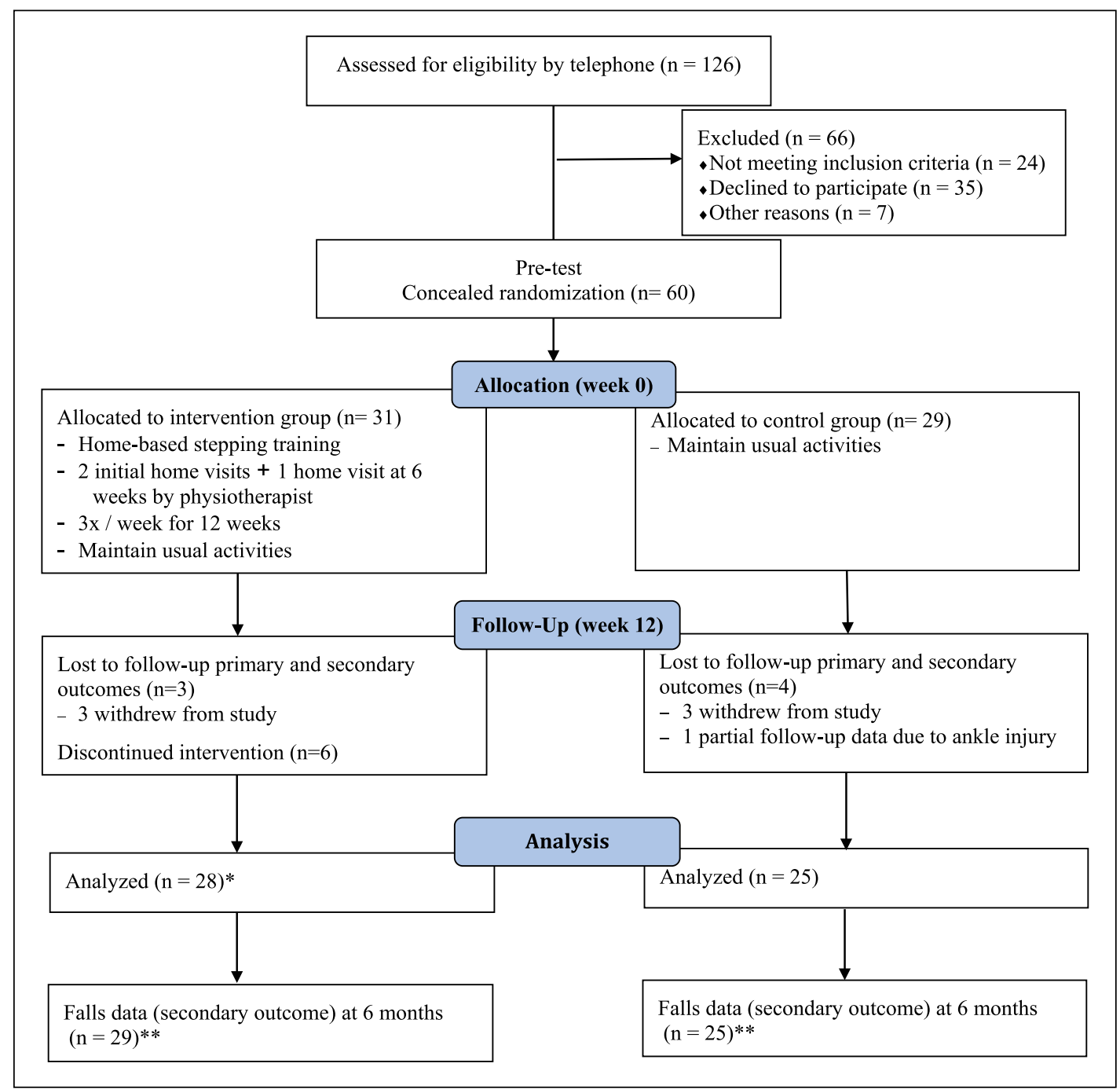

Figure I. Flow of participants through the trial.

*The six exercise participants who discontinued intervention include three participants lost to follow-up of both primary and secondary outcomes.

**Two participants in the exercise group and four participants in the control group had incomplete falls data.

discontinued training: two participants died, two participants ceased due to pre-existing lower back pain which was exacerbated by training, one participant sustained a knee injury due to a fall while walking to a car and one participant had family problems during the intervention period. Although eight participants' pre-existing pain (e.g. lower back pain, knee pain, foot pain) was exacerbated during the intervention period, participants reported that the exacerbation was not attributable to the stepping exercise. One participant reported a non-injurious fall while undertaking the exergame step training.

Of the 25 participants who completed the 12-week intervention, 18 participants progressed to the hard level, four participants progressed to the medium level and three participants remained at the 
Table I. Mean (SD) or number (\%) for participants' characteristics at baseline.

\begin{tabular}{lcc}
\hline Characteristic & \multicolumn{2}{l}{ Groups } \\
\cline { 2 - 3 } & Intervention $(n=31)$ & Control $(n=29)$ \\
\hline Age (years) & $68(7)$ & $65(7)$ \\
Gender (male) & $15(48 \%)$ & $9(31 \%)$ \\
Height (m) & $1.7(0.1)$ & $1.7(0.1)$ \\
Weight (kg) & $76(15)$ & $78(18)$ \\
Cognitive status & $28(2)$ & $29(1)$ \\
MMSE (0-30) & $7(4)$ & $9(6)$ \\
Duration of disease (years) & $31(11)$ & $33(13)$ \\
Disease severity & & $16(55 \%)$ \\
"On" MDS-UPDRS Part III (0-132) & $17(55 \%)$ & $7(24 \%)$ \\
Fallen in the past year (participants-yes) & $12(39 \%)$ & $757(498)$ \\
Freezing of gait (participants-yes) & $668(405)$ & \\
Daily levodopa equivalent dose (mg) & &
\end{tabular}

MMSE: Mini-Mental State Examination Score, MDS-UPDRS: Movement Disorders Society version of the Unified Parkinson's Disease Rating Scale.

easy level. Of the three participants who did not progress beyond the easy level, one participant found the novice level of the exergame too difficult initially. This participant began by standing on the mat and stepping in response to randomised instructions provided by a family member and progressed to the easy level by the end of the programme.

\section{Effect of the home-based intervention}

Data for primary and secondary outcomes at baseline and 12 weeks for intervention and control groups are presented in Table 2. There were no between-group differences in any of the choice stepping reaction time test measures or in the Functional Gait Assessment. In addition, there were no between-group differences in measures of muscle power, gait adaptability, simple reaction time, cognition or concern about falling. However, there was a significant between-group difference at post-test in favour of the control group for the performance of the Timed Up and Go.

Participants in the intervention group perceived an overall improvement in their mobility (mean change: 0.4 ; SD: 1.6), while the control group reported a deterioration of mobility (mean change: -0.5 ; SD: 1.5 ), resulting in a significant between-group difference of
0.9 (95\% confidence interval: -1.8 to $-0.1, P=0.03$ ). There was no significant between-group difference in perceived change in balance following the intervention (between-group difference: $0.6,95 \%$ confidence interval: -1.4 to $0.1, P=0.09$ ).

Participants in the exercise group reported a total of 136 falls (median 1 fall per person, interquartile range (IQR): 0-5), whereas participants in the control group reported 107 falls (median 1 fall per person, IQR: 0-3) during the six-month fall monitoring period (Supplementary Table S1). There was no significant between-group difference in fall rate (incidence rate ratios $=0.93(95 \%$ confidence interval: $0.33-2.57), P=0.88)$.

\section{Post hoc analysis}

There was a significant interaction between group and disease severity for reaction time $(P=0.02)$, and total response time $(P=0.01)$ during the choice stepping reaction time test and trends towards significance for power at low load $(P=0.08)$, Timed Up and Go test $(P=0.06)$ and hand reaction time $(P=0.05)$. The pattern of the interaction effects and the results of the subgroup analyses (Table 3, Supplementary Table S2) suggest a differential impact of the intervention according to disease 


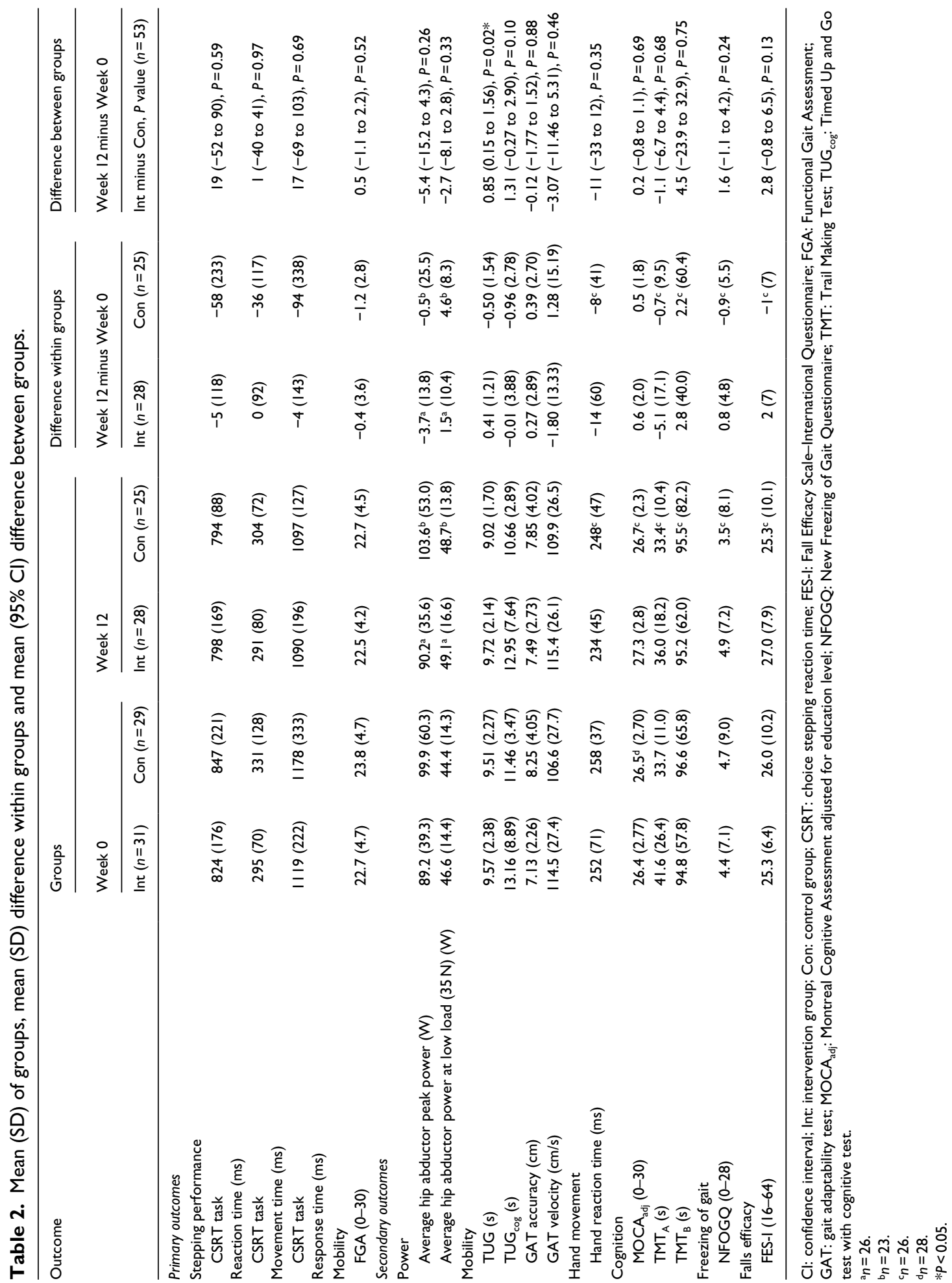


Table 3. Interactions for physical function variables.

\begin{tabular}{|c|c|c|c|c|}
\hline Variable & $\begin{array}{l}\text { Interaction } \\
P \text { value }\end{array}$ & Severity & $\begin{array}{l}\text { Subgroup } \\
P \text { value }\end{array}$ & Interpretation \\
\hline \multirow[t]{2}{*}{$\begin{array}{l}\text { CSRT Task } \\
\text { Reaction time }\end{array}$} & \multirow[t]{2}{*}{$0.02 *$} & High & $P=0.09$ & $\begin{array}{l}\text { Trend towards poorer scores } \\
\text { in intervention group }\end{array}$ \\
\hline & & Low & $P=0.07$ & $\begin{array}{l}\text { Trend towards improved scores } \\
\text { in intervention group }\end{array}$ \\
\hline $\begin{array}{l}\text { CSRT Task } \\
\text { Movement time }\end{array}$ & 0.24 & & & \\
\hline \multirow[t]{2}{*}{$\begin{array}{l}\text { CSRT Task } \\
\text { Response time }\end{array}$} & \multirow[t]{2}{*}{$0.01 *$} & High & $P=0.07$ & $\begin{array}{l}\text { Trend towards poorer scores } \\
\text { in intervention group }\end{array}$ \\
\hline & & Low & $P=0.12$ & $\begin{array}{l}\text { Trend towards improved scores } \\
\text { in intervention group }\end{array}$ \\
\hline FGA & 0.27 & & & \\
\hline $\begin{array}{l}\text { Average hip abductor peak } \\
\text { muscle power }\end{array}$ & 0.20 & & & \\
\hline \multirow[t]{2}{*}{$\begin{array}{l}\text { Average hip abductor power } \\
\text { at low load }(35 N)\end{array}$} & \multirow[t]{2}{*}{0.08} & High & $P=0.03 *$ & $\begin{array}{l}\text { Poorer scores in intervention } \\
\text { group }\end{array}$ \\
\hline & & Low & $P=0.65$ & \\
\hline \multirow[t]{2}{*}{ TUG } & \multirow[t]{2}{*}{0.055} & High & $P=0.002 *$ & $\begin{array}{l}\text { Poorer scores in intervention } \\
\text { group }\end{array}$ \\
\hline & & Low & $P=0.83$ & \\
\hline \multirow[t]{2}{*}{ Hand reaction time } & \multirow[t]{2}{*}{0.051} & High & $P=0.47$ & \\
\hline & & Low & $P=0.01 *$ & $\begin{array}{l}\text { Improved scores in intervention } \\
\text { group }\end{array}$ \\
\hline
\end{tabular}

CSRT: choice stepping reaction time; FGA: Functional Gait Assessment; TUG: Timed Up and Go test. $* P<0.05$.

severity with seemingly positive effects for the lowseverity group and potentially negative effects for the high-severity group.

\section{Discussion}

This randomised controlled trial showed that compared to no intervention, 12 weeks of minimally supervised home-based exergame step training was not effective in improving stepping performance, mobility, muscle power, cognition, simple reaction time, freezing of gait or fear of falling, or in reducing the number of falls in people with Parkinson's disease.

Our findings are in contrast to the results from similar home-based exergame interventions conducted in older people ${ }^{7}$ and in people with multiple sclerosis. ${ }^{10}$ The exercise programme in these studies consisted of exergame step training for two to three sessions per week and a choice stepping reaction time task once a week. It is possible that additional task-specific training using choice stepping reaction time task practice may have had a positive impact on stepping performance in the previous studies. Another possible explanation of the different results is the amount of practice. While the trial for older people prescribed 15- to 20-minute sessions, two to three times per week for 8 weeks, ${ }^{7}$ the trial for people with multiple sclerosis prescribed 30-minute sessions twice per week for 12 weeks. ${ }^{10}$ The present trial prescribed 15-minute sessions, three times per week for 12 weeks, meaning that the multiple sclerosis trial resulted in an overall higher dose of exercise. It is possible that people with a neurological condition may require a higher dose of stepping exercise than the general older population, ${ }^{28}$ and that the dose used in the present trial was inadequate. 
However, the post hoc subgroup analyses in the present trial also suggest that this form of step training may not be suitable for people with Parkinson's disease with higher disease severity. Therefore, further research is required to investigate the influence of both dose of training and disease severity on the efficacy of exergame-based step training in people with Parkinson's disease.

There were no improvements in peak and low load hip abductor muscle power following the exergame step training. Previous studies suggest that providing a load in addition to movement speed is the critical stimulus for improving muscle power. ${ }^{16}$ However, the exergame step training used in this study required participants to step as accurately as possible, in terms of both direction and timing, while synchronising their step responses to stimuli presented on the screen. Therefore, the lack of improvement in muscle power may be explained by the absence of an imperative for very fast movements as well as an absence of additional load.

A decrement in performance of the intervention group compared to the control group in the Timed Up and Go test is an unanticipated finding. This result could be explained by the emphasis on accurate, safe stepping in the exergame step training. As all participants were asked to perform the Timed $\mathrm{Up}$ and Go test at their comfortable and safe pace, the exercise group participants may have focused more on maintaining balance and performing the task safely at post-test, resulting in a longer time taken to complete the Timed Up and Go test. Exploration of the trend towards a differential effect of the intervention according to disease severity on the Timed Up and Go outcome shows that the high disease severity intervention group performing significantly worse on the Timed Up and Go post intervention compared to the high disease severity control group, while there was no apparent between-group difference for the low disease severity participants. Nonetheless, this finding should be interpreted with caution and could be attributable to a type 1 error.

The direction of interaction effects between intervention and disease severity was consistent across the physical function measures. This minimally supervised, home-based exergame intervention tended to have positive outcomes or no impact for lower disease severity participants, while the higher disease severity participants experienced no impact or potentially negative outcomes. While these results need to be interpreted with caution and replicated, previous studies have reported that home-based physiotherapy exercise programmes have a greater impact on reducing falls in people with lower Parkinson's disease severity compared to people with higher disease severity. ${ }^{24,29}$ In addition, a recent trial showed that the efficacy of home-based interventions with minimum supervision is likely to be reduced in the presence of comorbidities and suggested that exercise with supervision, either individually or in a group setting, may be critical to overcoming obstacles associated with higher Parkinson's disease severity. ${ }^{30}$ Taken together, it appears that minimally supervised home-based exercise programmes may be beneficial for people with lower Parkinson's disease severity, whereas people with higher Parkinson's disease severity may require additional supervision. Moreover, tailoring the intervention to specific deficits, progressing and adjusting the intervention at an adequate pace may be considered for people with higher Parkinson's disease severity who have a greater risk of comorbidities. Further research is required to determine whether increased levels of supervision and customisation of the intervention are more effective than providing the exergame in a minimally supervised format.

Not only were there no overall positive effects of the intervention on physical function, but also, there was also no effect on cognitive measures. This exergame step training requires divided and selective attention and executive function, ${ }^{4}$ so training effects may not generalise to other cognitive functions measured by the Montreal Cognitive Assessment ${ }^{20}$ and Trail Making Test parts A and B. ${ }^{21}$ In addition, the dose of the intervention may have been insufficient.

Improvements in self-reported mobility favoured the exergame step training group. Although it did not reach statistical significance, participants from the step training group also reported improvements in balance, whereas participants from the control group reported deterioration in balance. Similar results were found in a previous study measuring perceptions of a dance exergame programme in 
people with Parkinson's disease where participants reported that the exergame programme improved their balance and coordination. ${ }^{9}$ This result could be influenced by the inability to blind participants to their group allocation in the present trial. Notably, however, while these subjective improvements suggest the exercise participants increased their confidence in relation to their mobility, there was no corresponding improvement in their concern about falling as measured by the Falls Efficacy ScaleInternational. The fact that these subjective improvements were reported despite the lack of improvement in other outcome measures highlights the importance of evaluating individuals' perceptions of the efficacy of interventions.

One of the advantages of using exergames as therapeutic exercise is their potential to provide low-cost, pragmatic, sustainable and engaging exercise options. ${ }^{31}$ However, the evidence for efficacy is, to date, largely from trials conducted in fully supervised settings. $6,32-34$ The results from the present trial show that people with mild to moderate Parkinson's disease with sufficient cognition can participate in home-based exergame programmes. Due to the minimal supervision provided in the present trial, we utilised paper-based log books to facilitate and monitor adherence ${ }^{35}$ and participants reported a high level of adherence ( $86 \%$ of prescribed exercises) to the programme. This, along with the fact that most participants were able to progress their level of difficulty, suggests that this is a feasible form of exercise that is appealing to people with Parkinson's disease. Further investigation of minimally supervised home-based exergame programmes is therefore warranted.

There are several limitations to this study. First, the small sample size means that there was limited statistical power to find any effects of the intervention, and that the subgroup analysis should be interpreted with caution. Second, the intervention was delivered over a short time frame and we were unable to conduct any follow-up evaluation. As Parkinson's disease is a degenerative disorder, future research should consider the effects of exergames over the longer term. Finally, our sample comprised relatively high-functioning people with Parkinson's disease, so the findings cannot be generalised to people with advanced Parkinson's disease.
In conclusion, our results suggest that while minimally supervised home-based exergame step training did not show a positive effect on stepping performance or functional mobility in people with Parkinson's disease, participants perceived mobility benefits post training. This form of exercise was delivered with minimal supervision at home for people with mild Parkinson's disease, adherence to the programme was high and participants perceived that their mobility improved, suggesting that exergames may provide an engaging form of sustainable home-based exercise for people with Parkinson's disease. The differential effects of the programme according to disease severity suggest that this type of intervention delivered with minimal supervision may be most appropriate for people with lower disease severity. Future studies are needed to assess the long-term effects of homebased exergame programmes and further explore the impact of disease severity.

\section{Clinical Messages}

- Home-based exergame step training is a feasible form of exercise in people with Parkinson's disease without significant cognitive or balance impairment.

- Overall, home-based exergame step training did not improve stepping performance or gait.

- Such training may have some benefits for improving stepping strategies in people with lower disease severity.

\section{Declaration of Conflicting Interests}

The author(s) declared no potential conflicts of interest with respect to the research, authorship and/or publication of this article.

\section{Funding}

The author(s) disclosed receipt of the following financial support for the research, authorship, and/or publication of this article: This research was supported by a Parkinson's NSW Bendigo Bank Parkinson's Research Grant, and a University of Sydney Bridging Support Grant. C Sherrington and S Lord are supported by Australian National Health and Medical Research 
Council Senior Research Fellowships. MJD Caetano was supported by the Conselho Nacional de Desenvolvimento Científico e Tecnológico (CNPq-200748/ 2012-2).

\section{References}

1. Hely MA, Reid WG, Adena MA, et al. The Sydney multicenter study of Parkinson's disease: the inevitability of dementia at 20 years. Mov Disord 2008; 23: 837-844.

2. Strecker K and Schwarz J. Parkinson's disease: emerging pharmacotherapy. Expert Opin Emerg Drugs 2008; 13: 573-591.

3. Smulders K, Esselink RA, Bloem BR, et al. Freezing of gait in Parkinson's disease is related to impaired motor switching during stepping. Mov Disord 2015; 30: 1090 1097.

4. Kadivar Z, Corcos DM, Foto J, et al. Effect of step training and rhythmic auditory stimulation on functional performance in Parkinson patients. Neurorehabil Neural Repair 2011; 25: 626-635.

5. Mirelman A, Rochester L, Reelick M, et al. V-TIME: a treadmill training program augmented by virtual reality to decrease fall risk in older adults: study design of a randomized controlled trial. BMC Neurol 2013; 13: 15.

6. Mirelman A, Rochester L, Maidan I, et al. Addition of a non-immersive virtual reality component to treadmill training to reduce fall risk in older adults (V-TIME): a randomised controlled trial. Lancet 2016; 188: 1170-1182.

7. Schoene D, Lord SR, Delbaere K, et al. A randomized controlled pilot study of home-based step training in older people using videogame technology. PLOS ONE 2013; 8: e57734.

8. O'Brien C, Clemson L and Canning CG. Multiple factors, including non-motor impairments, influence decision making with regard to exercise participation in Parkinson's disease: a qualitative enquiry. Disabil Rehabil 2016; 38: 472-481.

9. Natbony LR, Zimmer A, Ivanco LS, et al. Perceptions of a videogame-based dance exercise program among individuals with Parkinson's disease. Games Health J 2013; 2: 235-239.

10. Hoang P, Schoene D, Gandevia S, et al. Effects of a homebased step training programme on balance, stepping, cognition and functional performance in people with multiple sclerosis - a randomized controlled trial. Mult Scler 2016; 22: 94-103.

11. Folstein M, Folstein S and McHugh P. "Mini-mental state." A practical method for grading the cognitive state of patients for the clinician. J Psychiatr Res 1975; 12: 189-198.

12. Goetz CG, Stebbins GT and Tilley BC. Calibration of unified Parkinson's disease rating scale scores to Movement Disorder Society-unified Parkinson's disease rating scale scores. Mov Disord 2012; 27: 1239-1242.

13. Lord SR and Fitzpatrick RC. Choice stepping reaction time: a composite measure of falls risk in older people. $J$ Gerontol A Biol Sci Med Sci 2001; 56: M627-M632.
14. Leddy AL, Crowner BE and Earhart GM. Functional gait assessment and balance evaluation system test: reliability, validity, sensitivity, and specificity for identifying individuals with Parkinson disease who fall. Phys Ther 2011; 91: 102-113.

15. Wrisley DM and Kumar NA. Functional gait assessment: concurrent, discriminative, and predictive validity in community-dwelling older adults. Phys Ther 2010; 90: 761-773.

16. Paul SS, Canning CG, Sherrington C, et al. Reduced muscle strength is the major determinant of reduced leg muscle power in Parkinson's disease. Parkinsonism Relat Disord 2012; 18: 974-977.

17. Morris S, Morris ME and Iansek R. Reliability of measurements obtained with the Timed "Up \& Go" test in people with Parkinson disease. Phys Ther 2001; 81: 810-818.

18. Caetano MJ, Menant JC, Schoene D, et al. Sensorimotor and cognitive predictors of impaired gait adaptability in older people. J Gerontol A Biol Sci Med Sci. Epub ahead of print 29 August 2016. DOI: 10.1093/gerona/glw171.

19. Lord SR, Menz HB and Tiedermann A. A physiological profile approach to falls risk assessment and prevention. Phys Ther 2003; 83: 237-252.

20. Nazem S, Siderowf AD, Duda JE, et al. Montreal cognitive assessment performance in patients with Parkinson's disease with "normal" global cognition according to minimental state examination score. J Am Geriatr Soc 2009; 57: 304-308.

21. Higginson CI, Lanni K, Sigvardt KA, et al. The contribution of trail making to the prediction of performance-based instrumental activities of daily living in Parkinson's disease without dementia. J Clin Exp Neuropsychol 2013; 35: 530-539.

22. Nieuwboer A, Rochester L, Herman T, et al. Reliability of the new freezing of gait questionnaire: agreement between patients with Parkinson's disease and their carers. Gait Posture 2009; 30: 459-463.

23. Yardley L, Beyer N, Hauer K, et al. Development and initial validation of the Falls Efficacy Scale-International (FES-I). Age Ageing 2005; 34: 614-619.

24. Canning CG, Sherrington C, Lord SR, et al. Exercise for falls prevention in Parkinson disease: a randomized controlled trial. Neurology 2015; 84: 304-312.

25. Kamper SJ, Maher CG and Mackay G. Global rating of change scales: a review of strengths and weaknesses and considerations for design. J Man Manip Ther 2009; 17: $163-170$

26. Paul SS, Canning CG, Song J, et al. Leg muscle power is enhanced by training in people with Parkinson's disease: a randomized controlled trial. Clin Rehabil 2014; 28 : 275-288.

27. Tomlinson CL, Stowe R, Patel S, et al. Systematic review of levodopa dose equivalency reporting in Parkinson's disease. Mov Disord 2010; 25: 2649-2653.

28. Foreman KB, Sondrup S, Dromey C, et al. The effects of practice on the concurrent performance of a speech and postural task in persons with Parkinson disease and healthy controls. Parkinsons Dis 2013; 2013: 987621.

29. Ashburn A, Fazakarley L, Ballinger C, et al. A randomised controlled trial of a home based exercise programme to 
reduce the risk of falling among people with Parkinson's disease. J Neurol Neurosurg Psychiatry 2007; 78: 678-684.

30. King LA, Wilhelm J, Chen Y, et al. Effects of group, individual, and home exercise in persons with Parkinson disease: a randomized clinical trial. J Neurol Phys Ther 2015; 39: 204-212.

31. Barry G, Galna B and Rochester L. The role of exergaming in Parkinson's disease rehabilitation: a systematic review of the evidence. J Neuroeng Rehabil 2014; 11: 33.

32. Mirelman A, Maidan I, Herman T, et al. Virtual reality for gait training: can it induce motor learning to enhance complex walking and reduce fall risk in patients with Parkinson's disease? J Gerontol A Biol Sci Med Sci 2011; 66: 234-240.
33. Pompeu JE, Arduini LA, Botelho AR, et al. Feasibility, safety and outcomes of playing Kinect Adventures! for people with Parkinson's disease: a pilot study. Physiotherapy 2014; 100: 162-168.

34. Van den Heuvel MR, Kwakkel G, Beek PJ, et al. Effects of augmented visual feedback during balance training in Parkinson's disease: a pilot randomized clinical trial. Parkinsonism Relat Disord 2014; 20: 1352-1358.

35. Allen NE, Sherrington C, Suriyarachchi GD, et al. Exercise and motor training in people with Parkinson's disease: a systematic review of participant characteristics, intervention delivery, retention rates, adherence, and adverse events in clinical trials. Parkinsons Dis 2012; 2012: 854328 (15 pp.). 